\title{
Distribution of Deep Gray Matter Lesions on Magnetic Resonance Imaging in Lymphomatosis Cerebri
}

\author{
Zen Kobayashi ${ }^{1}$, Sawako Sakai ${ }^{1}$, Sakiko Itaya ${ }^{2}$, Yoshiyuki Numasawa ${ }^{3}$, Kiyobumi Ota $^{4}$, \\ Miho Akaza ${ }^{5}$, Yasuhiro Ueda ${ }^{6}$, Shinichi Ogawa ${ }^{7}$, Shoichiro Ishihara ${ }^{1}$, \\ Hiroyuki Tomimitsu ${ }^{1}$ and Shuzo Shintani ${ }^{1}$
}

\begin{abstract}
:
We herein report the distribution of gray matter lesions on magnetic resonance imaging (MRI) in two patients with lymphomatosis cerebri (LC). In our patients, the fluid-attenuated inversion recovery sequence of brain MRI demonstrated a bilateral and diffuse high signal intensity, not only in the white matter but also in the thalamus, globus pallidus, putamen, and hippocampus. Among the deep gray matter, the caudate head and putamen (striatum) were relatively spared when compared with the globus pallidus, thalamus, and hippocampus. Interestingly, we found seven previous reports of similar MRI findings, with relative sparing of the striatum, in patients with LC. This finding may be characteristic of LC and help facilitate its diagnosis. Further investigations of a larger number of LC patients are necessary to confirm these findings.
\end{abstract}

Key words: caudate head, deep gray matter, lymphomatosis cerebri, putamen, striatum

(Intern Med 60: 623-627, 2021)

(DOI: 10.2169/internalmedicine.5200-20)

\section{Introduction}

Lymphomatosis cerebri (LC) is a rare form of primary central nervous system lymphoma (PCNSL) that predominantly involves the white matter. Pathological examinations of LC patients demonstrate the diffuse infiltration of lymphoma cells into the nerve fibers (1). Immunohistochemical studies have revealed diffuse large B-cell lymphoma in most LC patients (2). However, why LC patients present with diffuse infiltration without mass formation remains unclear.

On magnetic resonance imaging (MRI), LC is characterized by diffuse, non-enhancing infiltrative lesions without mass formation or mass effect (1-9). The lesions of LC are likely to be most clearly demonstrated with a fluidattenuated inversion recovery (FLAIR) sequence $(2,3)$. The lesions also show a high signal intensity on diffusionweighted imaging (DWI), and most of the lesions exhibit an increase in apparent diffusion coefficient (ADC) values on an ADC map (4), which may reflect the diffuse infiltration of non-cohesive malignant lymphoid cells (4). The lesions of LC are found in the cerebral white matter in $77.8 \%$, corpus callosum in $42.2 \%$, basal ganglia in $33.3 \%$, thalamus in $26.7 \%$, midbrain in $46.7 \%$, pons in $44.5 \%$, medulla oblongata in $31.1 \%$, cerebellum in $26.6 \%$, and spinal cord in $11.1 \%$ of patients (3). The cerebral cortices, cerebellar cortices, and cerebellar dentate nuclei are also rarely but occasionally involved (3).

However, the distribution of deep gray matter lesions on MRI characteristic of LC has not been reported. We herein report the distribution of deep gray matter lesions on the FLAIR sequence of MRI in two patients with LC and review the literature.

\footnotetext{
${ }^{1}$ Department of Neurology, JA Toride Medical Center, Japan, ${ }^{2}$ Department of Neurology, Kanto Central Hospital, Japan, ${ }^{3}$ Aozora Clinic, Japan, ${ }^{4}$ Department of Neurology, Nakatsu Municipal Hospital, Japan, ${ }^{5}$ Department of Neurology and Neurological Science, Tokyo Medical and Dental University, Japan, ${ }^{6}$ Department of Neurosurgery, JA Toride Medical Center, Japan and ${ }^{7}$ Department of Hematology, JA Toride Medical Center, Japan
}

Received: April 30, 2020; Accepted: August 4, 2020; Advance Publication by J-STAGE: September 30, 2020

Correspondence to Dr. Zen Kobayashi, kobayashiz112@yahoo.co.jp 

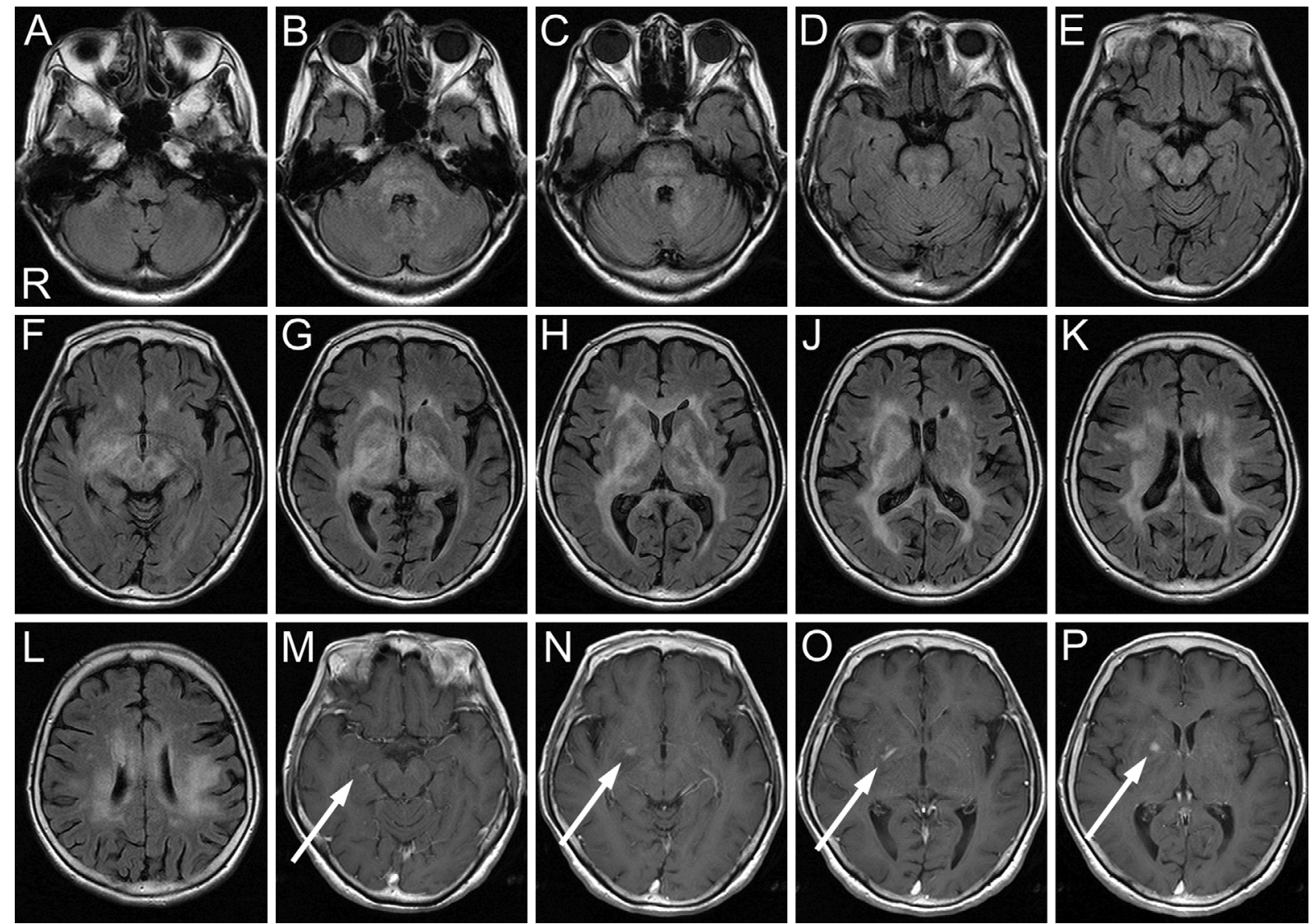

Figure 1. Fluid-attenuated inversion recovery (FLAIR) sequence of brain MRI (A-H, J-L) demonstrated a high signal intensity in the brainstem (A-F), left cerebellum (B, C), bilateral hippocampus $(D, E)$, bilateral thalamus $(G, H)$, bilateral globus pallidus $(G, H, J)$, bilateral putamen $(G, H, J)$, and bilateral cerebral white matter (F-H, J-L). The letter " $R$ " indicates the right side. The putamen and caudate head (striatum) were relatively spared. Gadolinium-enhanced T1-weighted image (GdT1WI) (M-P) showed abnormal enhancement from the left hippocampus (M, arrow) to the globus pallidus (N-P, arrow).

\section{Case Reports}

\section{Case 1}

A 67-year-old woman gradually presented with bradykinesia, followed by appetite loss and difficulty walking, and was admitted to our hospital about 1 month after the onset. She had no medical history of note. A neurological examination demonstrated a mask-like face, dysarthria, dysphagia, bradykinesia, muscle rigidity, frontal lobe sign, and pyramidal sign as well as a wide-based and short-stepped gate.

On MRI, bilateral brain lesions were most clearly demonstrated with a FLAIR sequence, which showed a high signal intensity in the brainstem, cerebellum, hippocampus, thalamus, putamen, globus pallidus, and cerebral white matter (Fig. 1). Among the deep gray matter, the caudate head and putamen (striatum) were relatively spared when compared with the globus pallidus, thalamus, and hippocampus. There was no apparent mass effect. On DWI, the lesions showed a faint high signal intensity. In an ADC map, most lesions exhibited a slight increase of the ADC values. A gadolinium- enhanced T1-weighted image (Gd-T1WI) showed abnormal enhancement from the right hippocampus to the globus pallidus (Fig. 1).

Blood tests demonstrated a soluble interleukin 2 receptor (sIL-2R) level of $264 \mathrm{U} / \mathrm{mL}$ (reference range: 145-519 U/ $\mathrm{mL}$ ). A lumbar puncture yielded watery clear cerebrospinal fluid (CSF). A CSF examination showed a cell count of 5/ $\mathrm{mm}^{3}$, total protein of $50 \mathrm{mg} / \mathrm{dL}$ (reference range: $8-43 \mathrm{mg}$ / $\mathrm{dL}$ ), glucose of $65 \mathrm{mg} / \mathrm{dL}$, sIL-2R of $63.1 \mathrm{U} / \mathrm{mL}$ (reference range: $<85$ ), and $\beta 2$ microglobulin of $5.50 \mathrm{mg} / \mathrm{L}$ (reference range: 0.69-1.29). Cytology of the CSF was negative.

A stereotactic brain biopsy of the right globus pallidus demonstrated the diffuse infiltration of large abnormal cells, which were immunopositive for CD20 and CD79a. A diagnosis of LC due to diffuse large B-cell lymphoma was established based on the MRI and biopsy findings.

\section{Case 2}

A 71-year-old man presented with progressive gait disorder and was admitted to our hospital about 5 months after the onset. His medical history included hypertension and chronic kidney disease. A neurological examination demon- 

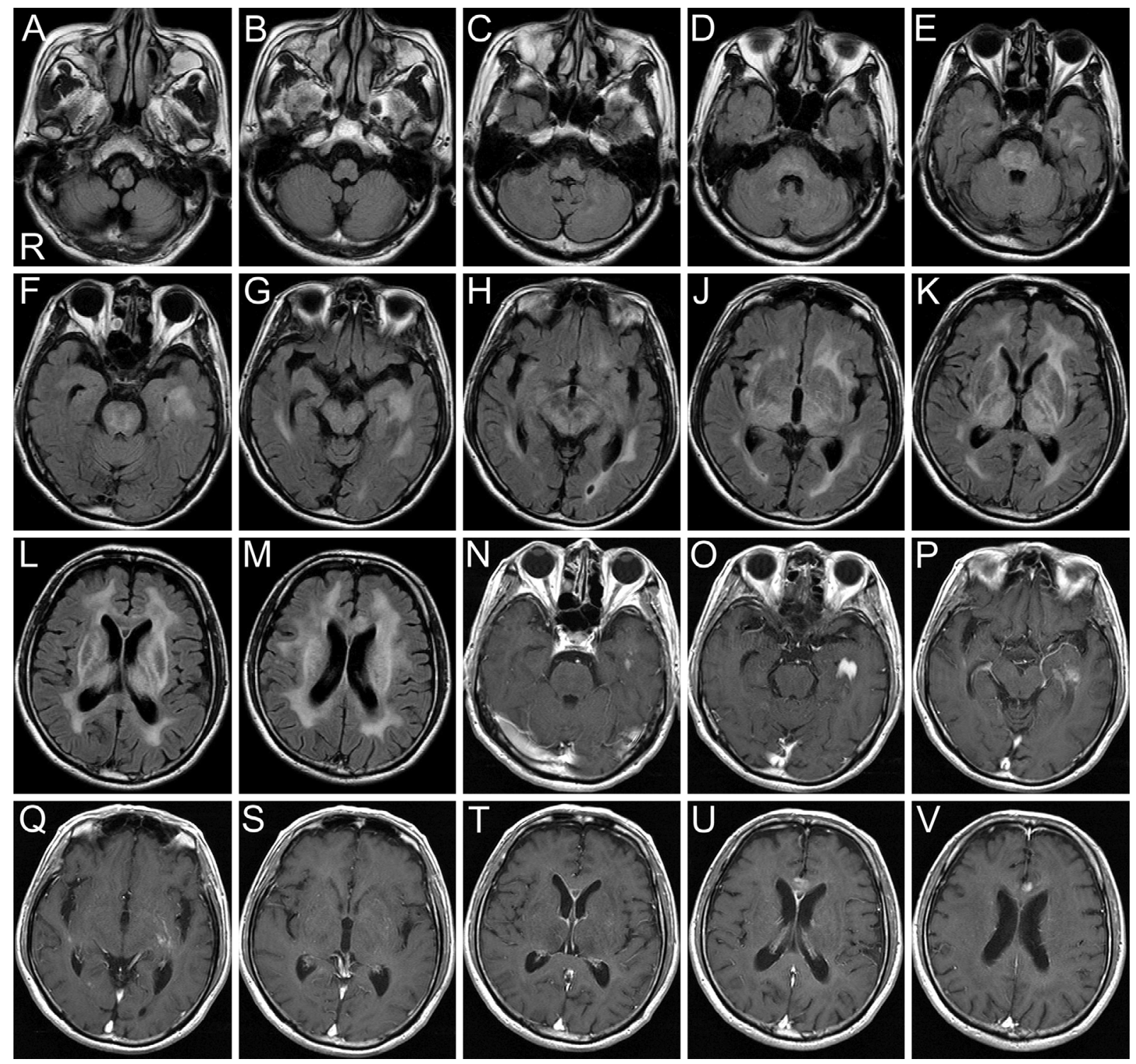

Figure 2. FLAIR sequence of brain MRI (A-H, J-M) demonstrated a high signal intensity in the brainstem (A-H), bilateral cerebellum (C, D), bilateral hippocampus (E-G), bilateral thalamus (J, K), bilateral caudate head $(\mathrm{K})$, bilateral globus pallidus $(\mathrm{K})$, bilateral putamen $(\mathrm{L})$, and bilateral cerebral white matter (E-H, J-M). The striatum was relatively spared. Gd-T1WI (N-Q, S-V) showed abnormal enhancement around the inferior horn of the left lateral ventricle (N-Q) and in the genu of the corpus callosum (T-V).

strated time-related disorientation, miscalculation, frontal lobe sign, and upper limb apraxia as well as limb and truncal ataxia.

On MRI, bilateral brain lesions were most clearly demonstrated with a FLAIR sequence, which showed a high signal intensity in the brainstem, cerebellum, hippocampus, thalamus, caudate head, putamen, globus pallidus, and cerebral white matter (Fig. 2). Among the deep gray matter, the caudate head and putamen (striatum) were relatively spared when compared with the globus pallidus, thalamus, and hippocampus. There was no apparent mass effect. Gd-T1WI showed abnormal enhancement around the inferior horn of the left lateral ventricle and in the corpus callosum (Fig. 2).

Blood tests showed that the sIL-2R level was slightly elevated to $723 \mathrm{U} / \mathrm{mL}$. A lumbar puncture yielded watery clear
CSF. The cell count was $9 / \mu \mathrm{L}$, comprising mononuclear cells of $8 / \mu \mathrm{L}$ and polymorphonuclear cells of $1 / \mu \mathrm{L}$. The total protein and glucose levels of the CSF were 51 and $61 \mathrm{mg} /$ $\mathrm{dL}$, respectively. The sIL-2R level of the CSF was elevated to $676 \mathrm{U} / \mathrm{mL}$. Cytology of the CSF was negative.

A stereotactic brain biopsy from the genu of the corpus callosum demonstrated the diffuse infiltration of large abnormal cells with an angiocentric pattern, which were immunopositive for CD10, CD20, and CD79a. A diagnosis of LC due to diffuse large B-cell lymphoma was established based on the MRI and biopsy findings.

\section{Discussion}

On MRI of our patients, the FLAIR sequence clearly 
Table. The Differential Diagnosis of Lymphomatosis Cerebri.

\begin{tabular}{|c|c|c|c|c|}
\hline & \multirow[b]{2}{*}{ Clinical course } & \multicolumn{3}{|c|}{ MRI findings } \\
\hline & & $\begin{array}{l}\text { Bilateral hemispheric } \\
\text { lesions on the first MRI } \\
\text { scan }\end{array}$ & Mass effect & $\begin{array}{l}\text { Relative sparing of the striatum } \\
\text { despite involvement of other } \\
\text { deep gray matter on FLAIR }\end{array}$ \\
\hline $\begin{array}{l}\text { Posterior reversible } \\
\text { encephalopathy syndrome }\end{array}$ & acute & $\begin{array}{c}\text { common } \\
\text { (posterior predominant) }\end{array}$ & none (or slight) & uncommon \\
\hline $\begin{array}{l}\text { Progressive multifocal } \\
\text { leukoencephalopathy }\end{array}$ & subacute & uncommon & none (or slight) & uncommon \\
\hline Lymphomatosis cerebri & subacute (or chronic) & common & none (or slight) & sometimes present \\
\hline Glioma & subacute (or chronic) & uncommon & present & uncommon \\
\hline
\end{tabular}

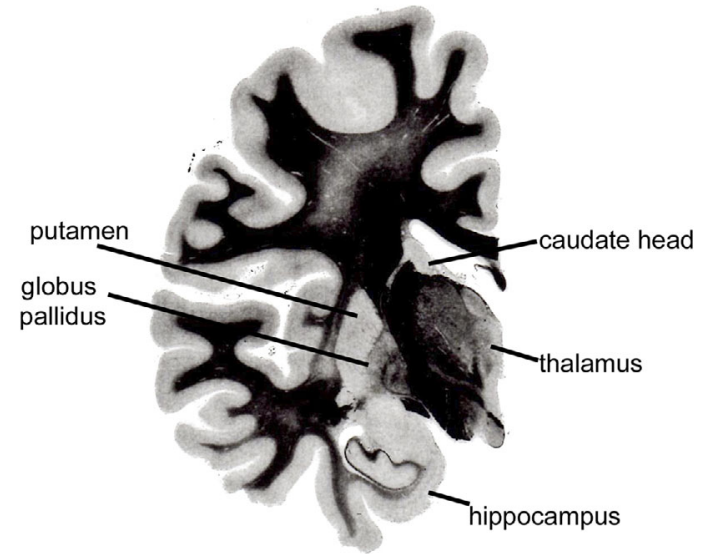

Figure 3. Klüver-Barrera staining (myelin staining) of a coronal section of the human postmortem brain hemisphere at the plane of the internal segment of the globus pallidus shows that the striatum is hardly stained in comparison with other deep gray matter tissues, including the globus pallidus, thalamus, and hippocampus.

demonstrated bilateral diffuse brain lesions, which showed no apparent mass effect. Enhancement of the lesions on GdT1WI was shown only in a small area. These MRI findings were consistent with LC. The differential diagnosis of LC is shown in Table. Izquierdo et al. reported that $95.2 \%$ of LC patients have bilateral hemispheric lesions on the first MRI scan (2), and this finding is uncommon in other diseases considered in the differential diagnosis (2).

In our patients, the caudate head and putamen (striatum) were relatively spared on MRI compared with the globus pallidus, thalamus, and hippocampus. Interestingly, we found seven previous reports of similar MRI findings with relative sparing of the striatum despite involvement of other deep gray matter in patients with LC $(2,3,5-9)$. This finding may be due to the fact that the nerve fibers are sparse in the striatum when compared with other deep gray matter. Fig. 3 shows the Klüver-Barrera staining (myelin staining) of a coronal section of the human postmortem brain hemisphere at the plane of the internal segment of the globus pallidus (an autopsy of this another patient was done at our hospital). This figure shows that the striatum is hardly stained compared with other deep gray matter tissues, in- cluding the globus pallidus, thalamus, and hippocampus.

In conclusion, in addition to the finding of "bilateral hemispheric lesions on the first MRI scan (2)", "relative sparing of the striatum despite involvement of other deep gray matter on FLAIR" may be characteristic of LC and provide a clue for the diagnosis of LC, although the absence of these findings does not necessarily exclude the possibility of LC. Further investigations of a larger number of LC patients are necessary to confirm these findings.

The authors state that they have no Conflict of Interest (COI).

\section{Acknowledgement}

The authors thank Takami Itoh, Yukinori Inadome, and Daisuke Kobayashi for contributing to the diagnosis and medical care of the patients.

\section{References}

1. Bakshi R, Mazziotta JC, Mischel PS, Jahan R, Seligson DB, Vinters HV. Lymphomatosis cerebri presenting as a rapidly progressive dementia: clinical, neuroimaging and pathologic findings. Dement Geriatr Cogn Disord 10: 152-157, 1999.

2. Izquierdo C, Velasco R, Vidal N, et al. Lymphomatosis cerebri: a rare form of primary central nervous system lymphoma. Analysis of 7 cases and systematic review of the literature. Neuro Oncol 18: 707-715, 2016

3. Li L, Rong JH, Feng J. Neuroradiological features of lymphomatosis cerebri: a systematic review of the English literature with a new case report. Oncol Lett 16: 1463-1474, 2018.

4. Yu H, Gao B, Liu J, et al. Lymphomatosis cerebri: a rare variant of primary central nervous system lymphoma and MR imaging features. Cancer Imaging 17: 26, 2017.

5. Raz E, Tinelli E, Antonelli M, et al. MRI findings in lymphomatosis cerebri: description of a case and revision of the literature. $\mathrm{J}$ Neuroimaging 21: e183-e186, 2011.

6. Imperiale D, Taraglio S, Atzori C, Testi R. Diffuse leukoencephalopathy due to lymphomatosis cerebri: a clinicopathological report. Neurol Sci 36: 1071-1073, 2015.

7. Kitai R, Hashimoto N, Yamate K, et al. Lymphomatosis cerebri: clinical characteristics, neuroimaging, and pathological findings. Brain Tumor Pathol 29: 47-53, 2012.

8. Yamaguchi $N$, Matsuda $S$, Yoshizawa $T$, Shigeeda R, Inoue $T$, Uchibori Y. A case of lymphomatosis cerebri rapidly confirmed by brain biopsy. Rinsho Shinkeigaku (Clin Neurol) 59: 286-289, 2019 (in Japanese, Abstract in English).

9. Watanabe M, Satoi H, Takahashi Y, Nishida N, Toda H, Matsumoto S. Remission of lymphomatosis cerebri induced by 
corticosteroid and high-doses intravenous methotrexate. Rinsho Shinkeigaku (Clin Neurol) 52: 486-490, 2012 (in Japanese, Abstract in English).
The Internal Medicine is an Open Access journal distributed under the Creative Commons Attribution-NonCommercial-NoDerivatives 4.0 International License. To view the details of this license, please visit (https://creativecommons.org/licenses/ by-nc-nd/4.0/).

(C) 2021 The Japanese Society of Internal Medicine

Intern Med 60: 623-627, 2021 